\title{
Recubrimiento a base de quitosano y extracto acuoso de hoja de Moringa oleífera obtenido por UMAE y su efecto en las propiedades fisicoquímicas de fresa (Fragaria $x$ ananassa)
}

\author{
Coating based on chitosan and aqueous extract of Moringa oleifera leaf obtained by UMAE and its effect \\ on the physicochemical properties of strawberry (Fragaria $x$ ananassa)
}

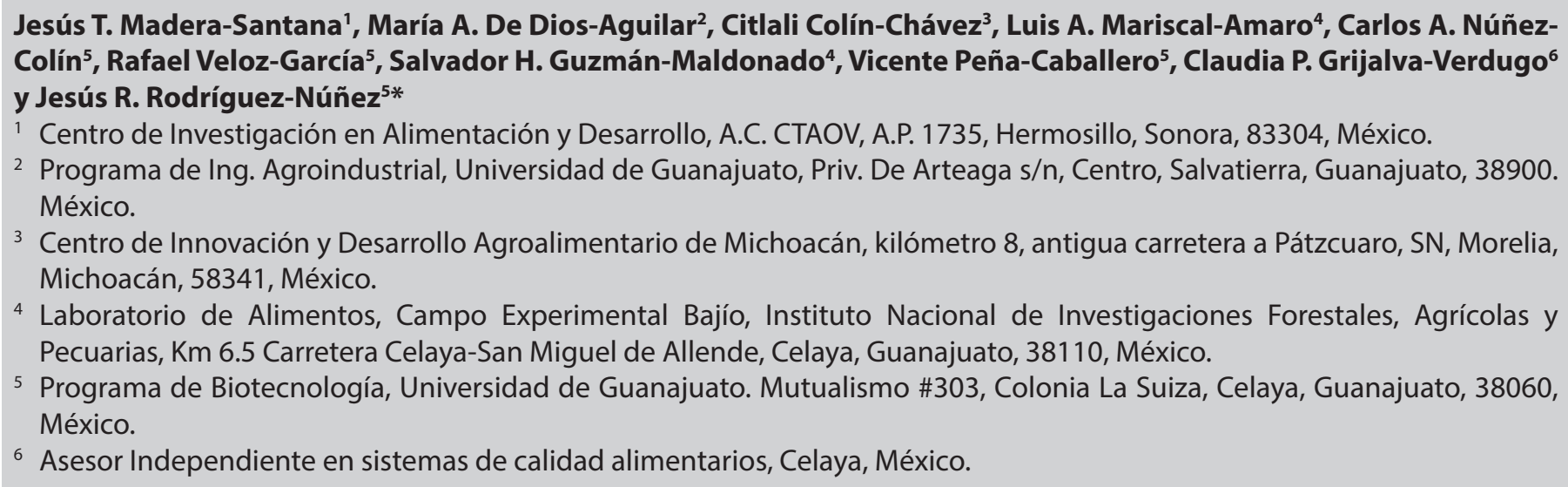

\section{RESUMEN}

Las fresas son frutos con una vida de anaquel corta debido a su elevada pérdida de humedad y a la sensibilidad al ataque de microorganismos. El objetivo del presente trabajo fue evaluar el efecto de los recubrimientos de quitosano adicionados con el extracto acuoso de hoja de Moringa oleifera en las propiedades fisicoquímicas. El extracto acuoso de hoja de Moringa se obtuvo mediante el método de extracción por ultrasonificación y microondas asistida. Se ensayaron dos formulaciones como recubrimientos y un control. Los parámetros evaluados fueron; pérdida de peso, $\mathrm{pH}$, acidez titulable, sólidos solubles totales, índice de sabor y color (Hue, L* y croma). El método de extracción por ultrasonido y microondas es eficaz para evitar el uso de solventes y reducir el tiempo de extracción. Los recubrimientos aplicados mejoraron el índice de sabor y disminuyeron los valores de acidez titulable (AT) mejorando así, la dulzura de la fresa.

Palabras clave: Microondas, ultrasonido, fenoles, fitoquímicos, extracción por ultrasonido y microondas asistida (UMAE).

\section{ABSTRACT}

Strawberries are fruits with a short shelf life due to their high loss of moisture, and susceptibility to microorganisms attack. The objective of the present work was to evaluate the effect of chitosan coatings added with Moringa oleifera extract, on the strawberries physicochemical properties. The aqueous extract of Moringa leaf was obtained by means of the ultrasonic extraction and assisted microwave method. Two formulations were tested as coatings and one control. The parameters evaluated were; weight loss, $\mathrm{pH}$, titratable acidity, total soluble solids, taste and color index (Hue, L *

*Autor para correspondencia: Jesús R. Rodríguez-Núñez

Correo electrónico: jesus.rodríguez@ugto.mx

Recibido: 11 de septiembre de 2018

Aceptado: 23 de enero de 2019 and chroma). The method of extraction by ultrasound and microwaves is effective to avoid the use of solvents and to reduce the time of extraction. The applied coatings improved the flavor index and decreased TA values thus improving the sweetness of the cutter.

Key words: Microwave, Ultrasound, Phenols, Phytochemicals, Ultrasound and Microwave Assisted Extraction (UMAE).

\section{INTRODUCCIÓN}

El cultivo de fresa en México reviste de gran importancia desde el punto de vista socioeconómico; según el servicio de Información Agroalimentaria y Pesquera (SIAP) de la SAGARPA en 2017 en la República Mexicana se sembraron 437,855 ton y las principales entidades productoras fueron Michoacán, Baja California, Guanajuato, Baja California Sur y Aguascalientes (SAGARPA, 2017).

Se ha reportado que las fresas son frutos no climatéricos muy delicados y con una vida de anaquel corta (Restrepo y Aristizábal, 2010), presentan elevada perdida de humedad y son sensibles al ataque de microorganismos, afectando sus atributos de sabor, olor, aroma y textura, y su valor comercial. Actualmente, el uso de recubrimientos comestibles a base de lípidos, polisacáridos y proteínas representa una alternativa viable para la conservación de frutas y hortalizas (GonzálezAguilar et al., 2009). Los cuales han sido aceptados debido a que crean una atmósfera modificada en la fruta, mediante el control de intercambio de gases respiratorios (Chien et al., 2007).

Entre los biopolímeros más utilizados para el recubrimiento de frutas y hortalizas se encuentra el quitosano, este biopolímero se obtiene a partir de la quitina mediante un proceso de desacetilación y está conformado por subu- 
nidades de $\mathrm{D}$-glucosamina y $\mathrm{N}$-acetyl-D-glucosamina ligadas mediante enlaces $\beta$ (1-4) (Chien et al., 2007). Entre las propiedades del quitosano se encuentran su toxicidad nula, buena maleabilidad, biocompatibilidad, su carácter antimicrobiano y antifúngico (Rodríguez-Núñez et al., 2012). Se ha reportado la aplicación en fresa de biopelículas de quitosano para recubrimientos de frutas como agente estructural semipermeable al $\mathrm{CO}_{2^{\prime}} \mathrm{O}_{2^{\prime}}$ y agua (López-Mata et al., 2012). Además, se ha demostrado la actividad fungicida del quitosano contra diversos patógenos que afectan los frutos durante su vida postcosecha, esto gracias a su efecto sobre el crecimiento micelial, esporulación, germinación y daño en hifas (Dutta et al., 2008; Zhang y Quantick, 2007; Jiang y Li, 2001).

La bio-compatibilidad del quitosano permite la adición de compuestos para la elaboración de bio-películas potencializadas con agentes antimicrobianos y antioxidantes extraídos de especies vegetales, principalmente (López-Mata et al., 2012). En este sentido, los extractos de hojas y semillas obtenidos del árbol de Moringa oleífera han sido reportados por su capacidad antimicrobiana y antifúngica en aplicaciones dérmicas (Chuang et al., 2007). En años recientes los extractos de hojas han sido utilizados en China y Taiwan como medicina herbolaria para el tratamiento del pie de atleta y Tinea cruris debido a su carácter antifúngico (Oliveira et al., 2011).

Los métodos convencionales para la extracción de compuestos vegetales utilizan solventes orgánicos como acetona, éter de petróleo y hexano, los cuales requieren largos periodos de tiempo, temperaturas elevadas, provocan la degradación de compuestos activos, además de resultar caros y generar residuos contaminantes (Chen et al., 2010; Lianfu y Zelong, 2008). El uso del equipo de extracción por ultrasonido y microondas asistida (UMAE, por sus siglas en inglés), se muestra como una buena alternativa para la obtención de extractos con degradación mínima de compuestos bioactivos como licopeno (Zhang y Zelong, 2008), quercetina (Huang et al., 2008) y aceites esenciales ricos en fitoquímicos (Wang et al., 2018), además, reduce el tiempo de extracción a segundos o minutos y mejora los rendimientos. Esto se debe a que la radiación no ionizante (microondas) no provoca la ruptura de los enlaces moleculares y no causa cambios en los compuestos por efecto de remoción de electrones. Además, al efecto del sonicado propaga ondas de sonido que crean cavitaciones en la solución, las cuales provocan la ruptura de burbujas de aire cerca de la superficie de la materia vegetal, induciendo la lisis celular (Liew et al., 2016).

Lianfu y Zelong (2008) reportaron la extracción de licopeno por UMAE, alcanzando una eficiencia de remoción del $97.4 \%$ en 6.1 min de tratamiento con $98 \mathrm{~W}$ en microondas y $40 \mathrm{kHz}$ sonicador. Liew et al. (2016) realizaron la obtención de pectinas de la cáscara de toronja mediante UMAE, alcanzando un rendimiento de $38 \%$ con 27.5 min de sonicado ( 40 $\mathrm{kHz}$ ) y 6.4 min de radiación en el microondas a $643.4 \mathrm{~W}$. En este sentido, la formulación de recubrimientos de quitosano potencializados con extractos acuosos de hojas de Moringa oleífera (EAHM) obtenidos por UMAE y su aplicación en fresas no ha sido evaluado, por lo cual, el objetivo del presente trabajo fue evaluar el efecto de los recubrimientos de quitosano adicionados con el extracto acuoso de hoja de Moringa oleífera obtenidos por UMAE en las propiedades fisicoquímicas de fresa (Fragaria x ananassa).

\section{MATERIALES Y MÉTODOS Recolección de las muestras}

Las muestras de hoja de Moringa oleifera fueron recolectadas de plantas localizadas en Empalme, Sonora, México. Las hojas fueron separadas de los peciolos y posteriormente se secaron en un horno (Felisa, FE-293A, México) a $60^{\circ} \mathrm{C}$ durante $12 \mathrm{~h}$. Posteriormente, se trituraron en un mortero y se almacenaron en bolsas Ziploc ${ }^{\oplus}$ en la oscuridad, hasta su uso. Se utilizaron fresas de la variedad Camarosa cultivadas en el municipio de Zamora, Michoacán. Las fresas se colectaron con un grado de madurez 5 según la Norma Oficial Mexicana NMX-FF-062-SCFI-200, presentando características de color homogéneas. Después de la cosecha, se almacenaron a $4^{\circ} \mathrm{C}$.

\section{Condiciones para la obtención de los EAHM}

LOS EAHM se realizaron en un equipo UMAE (X0-SM50, Nanjing, China). Se pesaron $7.5 \mathrm{~g}$ de hoja de Moringa oleifera y se añadieron $150 \mathrm{~mL}$ de agua tri-destilada $(1: 20 \mathrm{p} / \mathrm{v})$ en el reactor de $250 \mathrm{~mL}$ del equipo. Las condiciones de trabajo del sonicador y el microondas del equipo UMAE se programaron según la tabla 1. Con el fin de obtener 3 extractos a diferentes tiempos (10, 20 y $30 \mathrm{~min}$ ) los extractos obtenidos fueron filtrados al vacío utilizando filtros Whatman No. 41 y los sobrenadantes se almacenaron en frascos ámbar a $-4{ }^{\circ} \mathrm{C}$ hasta su uso, según el método propuesto por Dahmoune et al. (2015).

Tabla 1. Condiciones de trabajo programadas del equipo UMAE.

Table 1. Work conditions programmed for UMAE equipment.

\begin{tabular}{llll}
\hline \multicolumn{4}{c}{ Condiciones de sonicador } \\
\hline Potencia & $65 \mathrm{~W}(10 \%)$ \\
\hline Modo & $3(\theta 3)$ & \\
\hline Tiempo activado & $4 \mathrm{~s}$ & \\
\hline Tiempo desactivado & $2 \mathrm{~s}$ & \\
Condiciones de microondas & & & \\
Potencia de irradiación & $700 \mathrm{~W}$ & & \\
Tiempo & $10 \mathrm{~min}$ & $20 \mathrm{~min}$ & $30 \mathrm{~min}$ \\
\hline Temperatura registrada & $58.7^{\circ} \mathrm{C}$ & $69.1^{\circ} \mathrm{C}$ & $73.2^{\circ} \mathrm{C}$ \\
\hline
\end{tabular}

*Modo $3(\theta 3)$ indica el diámetro del sonicador utilizado $(6 \mathrm{~mm})$.

*Mode $3(\theta 3)$ indicates the diameter of the sonicator used $(6 \mathrm{~mm})$.

\section{Determinación cualitativa de fitoquímicos en los EAHM}

La determinación cualitativa de los fitoquímicos presentes en los EAHM se realizó mediante una batería de reacciones en placas 96 pozos. Las técnicas implementadas fueron: cloruro férrico (taninos y ácidos fenólicos), KillerKiliani (glucósidos), vainillina (taninos condensados), Meyer y Warner (alcaloides) y reactivo alcalino (flavonoides). Los resultados fueron registrados en base al cambio de color o formación de precipitados. Las metodologías usadas fueron propuestas por Dib et al. (2013), con algunas modificaciones. 


\section{Formulaciones y aplicación de los recubrimientos en fresa}

El quitosano utilizado para los recubrimientos fue obtenido de Sigma-Aldrich ${ }^{\circledR}$ (mediano peso molecular, 200-800 CP y grado de desacetilación de 75-78 \%). Las soluciones de quitosano usadas en los recubrimientos se prepararon al $2 \%$ $(\mathrm{p} / \mathrm{v})$ de quitosano disuelto en ácido acético al 1\% (v/v). La disolución del quitosano se realizó con agitador magnético durante $6 \mathrm{~h}$ a $150 \mathrm{rpm}$ a $25^{\circ} \mathrm{C}$. El tratamiento con EAHM se realizó agregando $1 \%$ de extracto a la solución de quitosano previamente preparado y se homogenizó en un ULTRATURRAX ${ }^{\circledR}$ T50 (IKA-Labortechni, Wilmington, USA) durante 2 min a 20,000 rpm a temperatura ambiente (Rodríguez-Núñez et al., 2012), en total se probaron 3 tratamientos: T1) quitosano puro al $2 \%$, T2) quitosano (2\%) + EAHM (1\%) y T3) control (sin recubrimiento).

Las fresas frescas fueron desinfectadas con una solución de cloro al 1\% durante $2 \mathrm{~min}$, posteriormente se enjuagaron y secaron con aire frio durante 5 min usando una secadora CONAIR $^{\circledR}$ 1875. Las fresas fueron recubiertas de forma manual con una brocha para repostería (silicón Prinz de $4 \mathrm{~cm}$ ) y se secaron con una corriente de aire frio con una secadora CONAIR ${ }^{\circledR} 1875$ durante $10 \mathrm{~min}$. El peso de cada fresa fue registrado en una balanza analítica (OHAUS, EP612, Nueva Jersey, USA) y fueron colocadas en charolas de unicel etiquetadas con el tratamiento. Posteriormente, las charolas de cada tratamiento fueron almacenadas en un refrigerador (Torrey, VRD42, México, Querétaro) a $4^{\circ} \mathrm{C}$ durante los 10 días del ensayo.

\section{Fenoles totales (FT)}

Los análisis de fenoles totales se cuantificaron en los extractos obtenidos y en las fresas tratadas con los recubrimientos, siguiendo la metodología propuesta por Singleton y Rossi (1965). Una alícuota de $50 \mu \mathrm{L}$ de cada muestra fue diluida en $3 \mathrm{~mL}$ de agua destilada más $250 \mu \mathrm{L}$ del reactivo Folin-Ciocalteu concentrado. Se Mezcló y dejó reposar $8 \mathrm{~min}$, posteriormente se agregaron $750 \mu \mathrm{L}$ de $\mathrm{Na}_{2} \mathrm{CO}_{3}$ al $20 \%$, se aforó a $5 \mathrm{~mL}$ con agua destilada y se dejó reposar $2 \mathrm{~h}$ a temperatura ambiente. Finalmente, se registró la absorbancia a $765 \mathrm{~nm}$ en un espectrofotómetro UV/Vis $(\mathrm{HACH}, \mathrm{DR} 3900$, USA). Los resultados se expresaron en $\mu \mathrm{g}$ de ácido gálico/g peso seco y se calcularon en base a una curva estándar de $\mathrm{R}^{2}$ $=0.9954$ y una ecuación de $y=0.8538 x+0.005$.

\section{Pérdida de peso (PP)}

La pérdida de peso acumulada se determinó registrando la diferencia entre el peso inicial y final de las fresas durante el almacenamiento y se expresó en porcentaje. Los resultados para la pérdida de peso fueron la media de 5 réplicas $(n=5)$ por tratamiento.

\section{Propiedades fisicoquímicas}

El pH se registró de los extractos obtenidos de fresas molidas en un mortero usando un potenciómetro (MettlerToledo, S500 SevenExellenceTM pH/ion, Indonesia). El por- centaje de acidez titulable (AT) se determinó agregando $7.5 \mathrm{~g}$ de fresa molida (utilizada para medir $\mathrm{pH}$ ) en $100 \mathrm{~mL}$ de agua tridestilada, se filtró al vacío usando filtros whatman No. 41 para obtener una alícuota de $25 \mathrm{~mL}$. Finalmente se tituló con una solución valorada de $\mathrm{NaOH} 0.1 \mathrm{~N}$ hasta alcanzar el pH 8.1 utilizando fenolftaleína al 1\% como indicador. Los resultados se expresaron como ácido málico (g de ácido málico/100 g) (AOAC, 1984).

Los sólidos solubles totales (SST) se midieron a partir de los índices de refracción usando un refractómetro (ATAGO, $\mathrm{RX}$-50000i-Plus, Tokio, Japón) a $20^{\circ} \mathrm{C}$ y los resultados se expresaron en porcentaje (AOAC, 1984). Además, se determinó el índice de sabor (IS) como el cociente SST sobre AT (AliaTejacal et al., 2012).

\section{Determinación de color}

Los cambios en el color característico (luminosidad $\left(L^{*}\right)$, ángulo Hue $\left(h^{*}\right)$ y cromaticidad $\left(C^{*}\right)$ ) se evaluaron usando un colorímetro (KONIKA MINOLTA ${ }^{\circledR}$, CR-410, Alemania), midiendo la epidermis del fruto. El ensayo se realizó según la metodología propuesta por la Rodríguez-Núñez et al. (2012), con algunas modificaciones. Donde $L^{*}$ es usada para determinar la luminosidad con rangos de 0 (negro) a 100 (transparente o blanco); $C^{*}$ representa la saturación del color y $h^{*}$ la tonalidad. La expresión de los resultados fue la media 10 frutos con 5 mediciones por fruto.

\section{Análisis estadístico}

Los análisis estadísticos fueron calculados utilizando SAS 9.3. Se aplicó un ANOVA en un diseño completamente al azar con medias repetidas en el tiempo, y un ANOVA en un diseño completamente al azar con una prueba de medias de Tukey $(P \leq 0.05)$ para cada evaluación. Este modelo de medias repetidas estima las diferencias entre las tendencias de los tratamientos en un periodo dado, así como los cambios dentro de cada tratamiento en el tiempo de evaluación y la posible interacción existente entre el tiempo y las tendencias de los tratamientos. A diferencia de un modelo de diseño de tratamientos factoriales, el tiempo, como fuente de variación, es un factor que repite la medición en la misma unidad experimental en diferentes días de evaluación por lo que tiene que considerarse dentro del modelo matemático (Cochran y Cox, 1995).

\section{RESULTADOS Y DISCUSIÓN Obtención del extracto acuoso de hoja de Moringa olei- fera}

La obtención del EAHM fue satisfactoria en tiempos cortos de 10 min alcanzando una temperatura máxima de $58.7{ }^{\circ} \mathrm{C}$, una potencia en microondas y $65 \mathrm{~W}$ de pulso de sonicado. El tiempo de $10 \mathrm{~min}$ fue seleccionado en base a los resultados obtenidos de fenoles totales y fitoquímicos, esto debido a que no se observaron diferencias significativas (DS) entre los 3 tiempos evaluados.

\section{Fenoles totales y perfil de fitoquímicos de EAHM}


Los contenidos de fenoles totales en los EAHM no mostraron DS $(18.83 \pm 1.0,16.09 \pm 1.7$ y $16.19 \pm 0.41 \mathrm{mg} / \mathrm{g})$ para los tiempos de extracción de 10, 20 y $30 \mathrm{~min}$, respectivamente. Los valores obtenidos coinciden con los rangos reportados por Guzmán-Maldonado et al. (2015), quienes señalan una concentración de fenoles totales en EAHM de 29-47 mg/g. Chumark et al. (2008) reportaron valores de $10 \mathrm{mg} / \mathrm{mL}$ de fenoles totales en EAHM, que es inferior a los valores reportados en la presente investigación.

Es necesario indicar que el uso de solventes puede mejorar la extracción de compuestos fenólicos en los EAHM, Moyo et al. (2012) reportaron fenoles totales en $120.33 \pm 0.76$ $\mathrm{mg} / \mathrm{g}$ en extractos de hojas de Moringa oleifera con acetona. La concentración de fenoles fue mayor debido a la diferencia de polaridades de los solventes usados para la extracción.

En relación al perfil de fitoquímicos en los EAHM se determinó la presencia de glucósidos cardiacos, taninos condensables, flavonoides y alcaloides (tabla 2). Estos resultados concuerdan con los reportados por diferentes autores como Verma et al. (2009) y Kasolo et al. (2010), quienes señalaron la presencia de flavonoides, ácidos fenólicos, alcaloides, taninos hidrolizables y condensados en EAHM.

Tabla 2. Fitoquímicos detectados mediante técnicas cualitativas en el EAHM.

Table 2. Phytochemicals detected by qualitative techniques in the EAHM.

\begin{tabular}{lcccc}
\hline Fitoquímico & Ensayo & $\begin{array}{c}\text { EAHM } \\
(\mathbf{1 0} \mathbf{~ m i n})\end{array}$ & $\begin{array}{c}\text { EAHM } \\
(\mathbf{2 0} \mathbf{~ m i n})\end{array}$ & $\begin{array}{c}\text { EAHM } \\
(\mathbf{3 0 ~} \mathbf{m i n})\end{array}$ \\
\hline $\begin{array}{l}\text { Taninos yácidos } \\
\text { fenólicos }\end{array}$ & $\begin{array}{l}\text { Cloruro } \\
\text { férrico }\end{array}$ & + & + & + \\
$\begin{array}{l}\text { Glucósidos } \\
\text { Killer-Kiliani }\end{array}$ & + & + & + \\
$\begin{array}{l}\text { Taninos } \\
\text { condensados }\end{array}$ & Vainillina & + & + & + \\
Alcaloides & Meyer & + & + & + \\
Alcaloides & Warner & + & + & + \\
Flavonoides & $\begin{array}{l}\text { Reactivo } \\
\text { alcalino }\end{array}$ & + & + & + \\
\hline
\end{tabular}

*Los signos indican: (+) presencia o (-) ausencia del fitoquímico.

\section{Pérdida de peso (PP)}

La fresa es un fruto que tiende a perder agua de forma rápida debido a su piel delgada provocando el encogimiento de la fruta y el ablandamiento del tejido (Velickova et al., 2013). En este sentido, los resultados referentes a PP no mostraron DS $(P \leq 0.05)$ entre los tratamientos en ninguno de los días evaluados (Tabla 3), así como en las tendencias de los mismos. Solamente se observó DS $(P \leq 0.05)$ entre los diferentes días de evaluación. La relación tratamiento $\mathrm{x}$ tiempo no presentó interacción significativa $(P \leq 0.05)$ (Tabla 4).

Los 3 tratamientos evaluados en el presente trabajo no contenían agentes hidrofóbicos en su composición, y es bien sabido que las películas de quitosano presentan un carácter hidrofílico lo cual no ayudó significativamente en la retención de humedad (Rodríguez-Núñez et al., 2014). Además, las características de las biopelículas obtenidas pueden variar en relación con las propiedades intrínsecas del quitosano (peso molecular y grado de desacetilación) (Suyatma et al., 2005) provocando diferencias entre las investigaciones realizadas.

Es recomendable añadir compuestos de naturaleza hidrofóbica (ácidos grasos, aceites esenciales, etc.) a las formulaciones de quitosano, esto debido a que incrementan el carácter hidrofóbico de los recubrimientos/biopelículas, disminuyendo la pérdida de humedad.

Tabla 4. Análisis de medias repetidas de la varianza en las propiedades fisicoquímicas de fresa recubiertas con quitosano y extractos de Moringa. Table 4. Analysis of repeated means of variance on the physicochemical properties of strawberry coated with chitosan and Moringa extracts.

\begin{tabular}{|c|c|c|c|c|c|c|c|}
\hline \multirow[b]{2}{*}{ Análisis } & \multicolumn{7}{|c|}{ F-Value } \\
\hline & PP & AT & SST & pH & IS & Hue & Croma L* \\
\hline Tratamiento & $0.86^{\mathrm{ND}}$ & $18.96^{* *}$ & $0.99^{N D}$ & $3.0^{\mathrm{ND}}$ & $24.03^{* *}$ & $1.28^{\mathrm{ND}}$ & $14.94^{* *} 1.47^{N D}$ \\
\hline Tiempo & $193.51^{* *}$ & $3.92^{* *}$ & $9.63^{* *}$ & $9.11^{* *}$ & $1.52^{\mathrm{ND}}$ & $4.46^{* *}$ & $4.67^{* *} 4.46^{* *}$ \\
\hline $\begin{array}{l}\text { Tratamiento } \\
\text { x tiempo }\end{array}$ & $0.87^{\mathrm{ND}}$ & $1.44^{\mathrm{ND}}$ & $1.38^{\mathrm{ND}}$ & $2.47^{* *}$ & $1.39^{\mathrm{ND}}$ & $1.65^{\mathrm{ND}}$ & $0.76^{\mathrm{ND}} 0.47^{\mathrm{ND}}$ \\
\hline
\end{tabular}

$*=0.05$ a $0.01 y^{* *}=<0.01$, existe DS. ND $=$ no existe $D S$.

Tabla 3. Efecto de los recubrimientos en los valores de PP (\%).

Table 3. Effect of coatings on the PP values (\%).

\begin{tabular}{lcccccccccc}
\hline & \multicolumn{10}{c}{ Día } \\
\cline { 2 - 10 } Tratamiento & $\mathbf{1}$ & $\mathbf{2}$ & $\mathbf{3}$ & $\mathbf{4}$ & $\mathbf{5}$ & $\mathbf{6}$ & $\mathbf{7}$ & $\mathbf{8}$ & $\mathbf{9}$ & $\mathbf{1 0}$ \\
\hline 1 & $0^{\text {ND }}$ & $1.233^{\mathrm{a}}$ & $2.096^{\mathrm{a}}$ & $3.001^{\mathrm{a}}$ & $3.621^{\mathrm{a}}$ & $4.218^{\mathrm{a}}$ & $5.140^{\mathrm{a}}$ & $5.940^{\mathrm{a}}$ & $6.549^{\mathrm{a}}$ & $7.490^{\mathrm{a}}$ \\
$\mathbf{2}$ & $0^{\text {ND }}$ & $1.525^{\mathrm{a}}$ & $2.612^{\mathrm{a}}$ & $3.699^{\mathrm{a}}$ & $4.670^{\mathrm{a}}$ & $5.420^{\mathrm{a}}$ & $6.590^{\mathrm{a}}$ & $7.095^{\mathrm{a}}$ & $7.810^{\mathrm{a}}$ & $8.644^{\mathrm{a}}$ \\
$\mathbf{3}$ & $0^{\text {ND }}$ & $3.490^{\mathrm{a}}$ & $4.663^{\mathrm{a}}$ & $5.630^{\mathrm{a}}$ & $6.621^{\mathrm{a}}$ & $7.207^{\mathrm{a}}$ & $8.004^{\mathrm{a}}$ & $8.485^{\mathrm{a}}$ & $8.993^{\mathrm{a}}$ & $9.828^{\mathrm{a}}$ \\
\hline
\end{tabular}

* Los superíndices con la misma letra en las columnas no muestran diferencia significativa $(P \leq 0.05)$ de acuerdo a la prueba de Tukey. ND representa el dato inicial por lo tanto no hay valores. T1) quitosano puro al 2\%, T2) quitosano (2\%) + EAHM (1\%) y T3) Control (sin recubrimiento). 


\section{Propiedades fisicoquímicas pH}

Los valores de $\mathrm{pH}$ mostraron $\mathrm{DS}(\mathrm{P} \leq 0.05)$ entre los tratamientos solo el día 9 , sin DS en el resto de los días, (Tabla 5). Los valores de $\mathrm{pH}$ en los tres tratamientos evaluados mostraron un aumento del $\mathrm{pH}$ con respecto al tiempo, sin embargo, los cambios no fueron significativos ( $P \leq 0.05)$. Además, se observó $D S(P \leq 0.05)$ entre los días de evaluación. La relación tratamiento $\mathrm{x}$ tiempo no presentó una interacción significativa $(P \leq 0.05)$ (tabla 4$)$.

Tabla 5. Efecto de los tratamientos en el pH de las fresas tratadas.

Table 5. Effect of treatments on the $\mathrm{pH}$ of treated strawberries.

\begin{tabular}{ccccccccccc}
\hline & \multicolumn{1}{c}{ Día } \\
\cline { 2 - 9 } Tratamiento & $\mathbf{1}$ & $\mathbf{2}$ & $\mathbf{3}$ & $\mathbf{4}$ & $\mathbf{5}$ & $\mathbf{6}$ & $\mathbf{7}$ & $\mathbf{8}$ & $\mathbf{9}$ & $\mathbf{1 0}$ \\
\hline $\mathbf{1}$ & $3.63^{\mathrm{a}}$ & $3.65^{\mathrm{a}}$ & $3.65^{\mathrm{a}}$ & $3.70^{\mathrm{a}}$ & $3.65^{\mathrm{a}}$ & $3.78^{\mathrm{a}}$ & $3.74^{\mathrm{a}}$ & $3.74^{\mathrm{a}}$ & $3.84^{\mathrm{a}}$ & $3.74^{\mathrm{a}}$ \\
$\mathbf{2}$ & $3.60^{\mathrm{a}}$ & $3.68^{\mathrm{a}}$ & $3.71^{\mathrm{a}}$ & $3.62^{\mathrm{a}}$ & $3.67^{\mathrm{a}}$ & $3.68^{\mathrm{a}}$ & $3.76^{\mathrm{a}}$ & $3.77^{\mathrm{a}}$ & $3.80^{\mathrm{a}}$ & $3.86^{\mathrm{a}}$ \\
$\mathbf{3}$ & $3.65^{\mathrm{a}}$ & $3.59^{\mathrm{a}}$ & $3.67^{\mathrm{a}}$ & $3.69^{\mathrm{a}}$ & $3.62^{\mathrm{a}}$ & $3.69^{\mathrm{a}}$ & $3.65^{\mathrm{a}}$ & $3.69^{\mathrm{a}}$ & $3.64^{\mathrm{b}}$ & $3.79^{\mathrm{a}}$ \\
\hline
\end{tabular}

* Los superíndices con la misma letra en las columnas no muestran diferencia significativa $(P \leq 0.05)$ de acuerdo a la prueba de Tukey. ND representa el dato inicial por lo tanto no hay valores. T1) quitosano puro al 2\%, T2) quitosano (2\%) + EAHM (1\%) y T3) Control (sin recubrimiento).

Duran et al. (2016) reportaron que no existe DS en los valores de $\mathrm{pH}$ en muestras de fresa recubiertas con quitosano en un ensayo de 40 días. Gol et al. (2013) mencionaron un aumento en el rango 3.96 a 4.16 de los valores de $\mathrm{pH}$ en fresas recubiertas (quitosano 1\% + HPMC 1\%) y no recubiertas (control) en un periodo de 12 días, siendo similar al rango encontrado en la presente investigación.

de Moraes et al. (2012) señalan que los recubrimientos comestibles en fresas retardan el aumento de $\mathrm{pH}$, fenómeno no observado en el presente trabajo, ya que no se obtuvo DS $(P<0.05)$ entre los tratamientos. Esto se puede relacionar con el tipo de recubrimiento utilizado, debido a que frecuentemente se realizan mezclas del quitosano con HPMC (Gol et al., 2013) o usan otro tipo de recubrimientos como alginatos y carrageninas (de Moraes et al., 2012).

\section{Acidez titulable (AT)}

Los resultados referentes a AT mostraron DS $(P \leq 0.05)$ entre los tratamientos solo el día 7, y sin DS en el resto de los días (tabla 6). Las tendencias de los tratamientos en el tiempo mostraron DS $(P \leq 0.05)$ por lo tanto, hubo cambios en los tratamientos a lo largo de los días presentándose pendientes positivas. Además, se mostró $D S(P \leq 0.05)$ entre los días de evaluación. La relación tratamiento $\mathrm{x}$ tiempo no presentó interacción significativa $(P \leq 0.05)$ (tabla 4).

Hernández-Muñoz et al. (2006) señalaron que existe DS en los valores de AT entre muestras de fresa recubiertas con quitosano y no recubiertas, observándose que los recubrimientos causan un decremento en los valores de AT debido a que disminuyen la respiración. Esto es consistente con lo encontrado en el presente trabajo, ya que los tratamientos con quitosano presentaron valores inferiores significativos $(P$ $\leq 0.05$ ) con respecto al control. Restrepo \& Aristizábal, (2010) señalan resultados similares en recubrimientos comestibles en fresa, y mencionan que tal disminución en los valores de acidez se debe a que se ralentiza la frecuencia respiratoria retrasando la utilización de los ácidos orgánicos en las reacciones enzimáticas.

\section{Sólidos solubles totales}

Los SST es un parámetro importante debido a que afecta la calidad del fruto y la aceptabilidad del consumidor (Aday y Caner., 2013). Los valores de SST mostraron DS (P $\leq$ 0.05 ) entre los tratamientos los días 1 y 7 , y sin DS en el resto de los días (tabla 7). Las tendencias de los tratamientos en el tiempo no mostraron DS $(P \leq 0.05)$, por lo tanto, no hubo cambios en los tratamientos en los días evaluados, presentándose pendientes positivas. Además, se observó DS $(\mathrm{P} \leq$ 0.05 ) entre los días de evaluación. La relación tratamiento $x$ tiempo presentó interacción significativa $(P \leq 0.05)$ (tabla 4).

Los resultados de SST son similares a los reportados por Gol et al. (2013) quienes señalaron que fresas recubiertas con biopelículas de quitosano 1\% + HPMC $1 \%$ presentan un aumento gradual en los valores de SST a los 8 días, y una posterior caída a los 12 días. Además, los presentes resultados difieren con los reportados por Duran et al. (2016) quienes mencionan una disminución de SST en los primeros 5 días de almacenamiento de fresas recubiertas con quitosano, mientras que las fresas no recubiertas presentaron una reducción drástica.

Aday y Caner (2013) reportaron que esta reducción en los valores de SST se debe a la degradación de la sacarosa, mientras que Velickova et al. (2013) mencionaron que este

Tabla 6. Efecto de los tratamientos sobre los valores de AT (\%).

Table 6. Effect of treatments on the AT values (\%).

\begin{tabular}{ccccccccccc}
\hline & \multicolumn{10}{c}{ Día } \\
\cline { 2 - 9 } Tratamiento & $\mathbf{1}$ & $\mathbf{2}$ & $\mathbf{3}$ & $\mathbf{4}$ & $\mathbf{5}$ & $\mathbf{6}$ & $\mathbf{7}$ & $\mathbf{8}$ & $\mathbf{9}$ & $\mathbf{1 0}$ \\
\hline $\mathbf{1}$ & $0.173^{\mathrm{a}}$ & $0.188^{\mathrm{a}}$ & $0.160^{\mathrm{a}}$ & $0.193^{\mathrm{a}}$ & $0.267^{\mathrm{a}}$ & $0.207^{\mathrm{a}}$ & $0.204^{\mathrm{ab}}$ & $0.204^{\mathrm{a}}$ & $0.204^{\mathrm{a}}$ & $0.182^{\mathrm{a}}$ \\
$\mathbf{2}$ & $0.166^{\mathrm{a}}$ & $0.145^{\mathrm{a}}$ & $0.172^{\mathrm{a}}$ & $0.159^{\mathrm{a}}$ & $0.167^{\mathrm{a}}$ & $0.182^{\mathrm{a}}$ & $0.173^{\mathrm{b}}$ & $0.185^{\mathrm{a}}$ & $0.187^{\mathrm{a}}$ & $0.173^{\mathrm{a}}$ \\
$\mathbf{3}$ & $0.179^{\mathrm{a}}$ & $0.179^{\mathrm{a}}$ & $0.173^{\mathrm{a}}$ & $0.208^{\mathrm{a}}$ & $0.216^{\mathrm{a}}$ & $0.213^{\mathrm{a}}$ & $0.239^{\mathrm{a}}$ & $0.213^{\mathrm{a}}$ & $0.230^{\mathrm{a}}$ & $0.207^{\mathrm{a}}$ \\
\hline
\end{tabular}

* Los superíndices con la misma letra en las columnas no muestran diferencia significativa $(\mathrm{P} \leq 0.05)$ de acuerdo a la prueba de Tukey. ND representa el dato inicial por lo tanto no hay valores. T1) quitosano puro al $2 \%$, T2) quitosano (2\%) + EAHM (1\%) y T3) Control (sin recubrimiento). 
Tabla 7. Efecto de los tratamientos sobre los valores de SST ( $\left.{ }^{\circ} \mathrm{Bx}\right)$. Table 7. Effect of treatments on the SST values ( $\left.{ }^{\circ} \mathrm{Bx}\right)$.

\begin{tabular}{cccccccccccc}
\hline & \multicolumn{10}{c}{ Día } \\
\cline { 2 - 11 } Tratamiento & $\mathbf{1}$ & $\mathbf{2}$ & $\mathbf{3}$ & $\mathbf{4}$ & $\mathbf{5}$ & $\mathbf{6}$ & $\mathbf{7}$ & $\mathbf{8}$ & $\mathbf{9}$ & $\mathbf{1 0}$ \\
\hline $\mathbf{1}$ & $9.922^{\mathrm{a}}$ & $10.827^{\mathrm{a}}$ & $10.33^{\mathrm{a}}$ & $10.622^{\mathrm{a}}$ & $11.221^{\mathrm{a}}$ & $11.22^{\mathrm{a}}$ & $12.201^{\mathrm{a}}$ & $12.991^{\mathrm{a}}$ & $12.213^{\mathrm{a}}$ & $13.218^{\mathrm{a}}$ \\
$\mathbf{2}$ & $9.036^{\mathrm{b}}$ & $9.475^{\mathrm{a}}$ & $10.341^{\mathrm{a}}$ & $9.977^{\mathrm{a}}$ & $11.936^{\mathrm{a}}$ & $11.936^{\mathrm{a}}$ & $12.835^{\mathrm{a}}$ & $12.458^{\mathrm{a}}$ & $12.429^{\mathrm{a}}$ & $11.866^{\mathrm{a}}$ \\
$\mathbf{3}$ & $9.374^{\mathrm{ab}}$ & $10.237^{\mathrm{a}}$ & $11.077^{\mathrm{a}}$ & $11.981^{\mathrm{a}}$ & $12.122^{\mathrm{a}}$ & $12.222^{\mathrm{a}}$ & $10.781^{\mathrm{b}}$ & $11.41^{\mathrm{a}}$ & $12.653^{\mathrm{a}}$ & $13.238^{\mathrm{a}}$ \\
\hline
\end{tabular}

*Los superíndices con la misma letra en las columnas no muestran diferencia significativa $(P \leq 0.05)$ de acuerdo a la prueba de Tukey. ND representa el dato inicial por lo tanto no hay valores. T1) quitosano puro al 2\%, T2) quitosano (2\%) + EAHM (1\%) y T3) Control (sin recubrimiento).

fenómeno en fresa es atribuido a la disminución de la respiración del fruto al final de la vida de anaquel. En el presente estudio no se observó una caída significativa de los valores de SST en el periodo de tiempo del ensayo, tal vez es necesario mantener el experimento por un periodo mayor de tiempo para observar este decremento, esto debido a que ninguno de los 3 tratamientos evaluados mostró DS en el rango de tiempo evaluado.

\section{Índice de sabor (SST/AT)}

Los resultados referentes al índice de sabor mostraron DS $(P \leq 0.05)$ entre los tratamientos los días 7 y 8 , y no se observó DS en el resto de los días (figura 8). Las tendencias de los tratamientos en el tiempo señalaron $\mathrm{DS}(\mathrm{P} \leq 0.05)$, mostrando cambios en los días de tratamiento, con pendientes positivas. Por otra parte, no se observó DS $(P \leq 0.05)$ entre los días de evaluación. La relación tratamiento $x$ tiempo presentó una interacción significativa $(P \leq 0.05)$ (tabla 4).

Los resultados obtenidos demuestran que las fresas recubiertas ( 1 y 2$)$ son significativamente $(P \leq 0.05)$ más dulces en comparación con las no recubiertas (control), esto según Ladaniya (2008), quien menciona que la relación entre SST/AT (índice de sabor) indica lo dulce o amargo de un fruto; así un incremento en el valor de la proporción corresponde a la disminución de la acidez. Alvarado et al. (2016) reportaron valores de SST y AT para cultivos de fresa bajo diferentes sistemas de producción (malla sombra blanca y negra) y es posible calcular valores de índice de sabor de 48.31 y 51.47, estos resultados están en el rango de los obtenidos en la presente investigación y se demuestra que bajo diferentes condiciones el índice de sabor sufre modificaciones.

\section{Color}

El comportamiento de los componentes del color se muestra en las tablas 9,10 y 11 . Con respecto al cambio en la tonalidad del color (Hue), las tendencias de los tratamientos en el tiempo no presentaron DS $(P \leq 0.05)$, por lo tanto, no se observaron cambios entre los tratamientos en los diferentes días de evaluación, obteniendo pendientes negativas, es decir, disminución en el color en los 3 tratamientos evalua-

Tabla 8. Efecto de los tratamientos en los valores de índice de sabor (IS). Table 8. Effect of treatments on the taste index values (IS).

\begin{tabular}{ccccccccccc}
\hline & \multicolumn{10}{c}{ Día } \\
\cline { 2 - 10 } Tratamiento & $\mathbf{1}$ & $\mathbf{2}$ & $\mathbf{3}$ & $\mathbf{4}$ & $\mathbf{5}$ & $\mathbf{6}$ & $\mathbf{7}$ & $\mathbf{8}$ & $\mathbf{9}$ & $\mathbf{1 0}$ \\
\hline $\mathbf{1}$ & $59.461^{\mathrm{a}}$ & $57.85^{\mathrm{a}}$ & $64.41^{\mathrm{a}}$ & $55.133^{\mathrm{a}}$ & $44.986^{\mathrm{a}}$ & $53.959^{\mathrm{a}}$ & $58.983^{\mathrm{b}}$ & $63.167^{\mathrm{ab}}$ & $59.971^{\mathrm{a}}$ & $72.718^{\mathrm{a}}$ \\
$\mathbf{2}$ & $52.514^{\mathrm{a}}$ & $71.57^{\mathrm{a}}$ & $60.29^{\mathrm{a}}$ & $62.726^{\mathrm{a}}$ & $71.139^{\mathrm{a}}$ & $65.548^{\mathrm{a}}$ & $73.997^{\mathrm{a}}$ & $67.491^{\mathrm{a}}$ & $67.564^{\mathrm{a}}$ & $70.612^{\mathrm{a}}$ \\
$\mathbf{3}$ & $52.37^{\mathrm{a}}$ & $57.25^{\mathrm{a}}$ & $63.79^{\mathrm{a}}$ & $58.63^{\mathrm{a}}$ & $56.23^{\mathrm{a}}$ & $56.972^{\mathrm{a}}$ & $45.333^{\mathrm{c}}$ & $53.619^{\mathrm{b}}$ & $54.762^{\mathrm{a}}$ & $63.806^{\mathrm{a}}$ \\
\hline
\end{tabular}

*Los superíndices con la misma letra en las columnas no muestran diferencia significativa $(P \leq 0.05)$ de acuerdo a la prueba de Tukey. ND representa el dato inicial por lo tanto no hay valores. T1) quitosano puro al $2 \%, \mathrm{~T} 2)$ quitosano $(2 \%)+$ EAHM (1\%) y T3) Control (sin recubrimiento).

Tabla 9. Efecto de los tratamientos en los valores de Hue.

Table 9. Effect of treatments on Hue values.

\begin{tabular}{cccccccccccc}
\hline & \multicolumn{10}{c}{ Día } \\
\cline { 2 - 10 } Tratamiento & $\mathbf{1}$ & $\mathbf{2}$ & $\mathbf{3}$ & $\mathbf{4}$ & $\mathbf{5}$ & $\mathbf{6}$ & $\mathbf{7}$ & $\mathbf{8}$ & $\mathbf{9}$ & $\mathbf{1 0}$ \\
\hline $\mathbf{1}$ & $36.593^{\mathrm{a}}$ & $29.583^{\mathrm{a}}$ & $29.59^{\mathrm{a}}$ & $31.067^{\mathrm{a}}$ & $31.54^{\mathrm{a}}$ & $28.517^{\mathrm{a}}$ & $29.07^{\mathrm{a}}$ & $29.283^{\mathrm{a}}$ & $28.7^{\mathrm{a}}$ & $30.797^{\mathrm{a}}$ \\
$\mathbf{2}$ & $38.863^{\mathrm{a}}$ & $38.093^{\mathrm{a}}$ & $35.657^{\mathrm{a}}$ & $36.13^{\mathrm{a}}$ & $37.527^{\mathrm{a}}$ & $38.313^{\mathrm{a}}$ & $38.793^{\mathrm{a}}$ & $39.06^{\mathrm{a}}$ & $35.77^{\mathrm{a}}$ & $33.387^{\mathrm{a}}$ \\
$\mathbf{3}$ & $34.773^{\mathrm{a}}$ & $33.997^{\mathrm{a}}$ & $32.833^{\mathrm{a}}$ & $33.11^{\mathrm{a}}$ & $32.37^{\mathrm{a}}$ & $33.343^{\mathrm{a}}$ & $30.9^{\mathrm{a}}$ & $30.22^{\mathrm{a}}$ & $28.3^{\mathrm{a}}$ & $25.187^{\mathrm{a}}$ \\
\hline
\end{tabular}

*Los superíndices con la misma letra en las columnas no muestran diferencia significativa $(P \leq 0.05)$ de acuerdo a la prueba de Tukey. ND representa el dato inicial por lo tanto no hay valores. T1) quitosano puro al $2 \%, \mathrm{~T} 2)$ quitosano $(2 \%)+$ EAHM (1\%) y T3) Control (sin recubrimiento). 
Tabla 10. Efecto de los tratamientos en la variable croma (saturación).

Table 10. Effect of treatments on the chroma variable (saturation).

\begin{tabular}{|c|c|c|c|c|c|c|c|c|c|c|}
\hline \multirow[b]{2}{*}{ Tratamiento } & \multicolumn{10}{|c|}{ Día } \\
\hline & 1 & 2 & 3 & 4 & 5 & 6 & 7 & 8 & 9 & 10 \\
\hline 1 & $90.757^{a}$ & $84.067^{\mathrm{a}}$ & $75.87^{b}$ & $79.363^{b}$ & $78.493^{b}$ & $74.077^{\mathrm{b}}$ & $69.867^{a}$ & $69.103^{b}$ & $74.297^{a}$ & $68.853^{a}$ \\
\hline 2 & $103.723^{a}$ & $94.893^{\mathrm{a}}$ & $93.587^{a}$ & $93.23^{\mathrm{ab}}$ & $98.107^{a}$ & $102.313^{\mathrm{a}}$ & $94.83^{\mathrm{a}}$ & $94.663^{a}$ & $85.827^{a}$ & $83.857^{\mathrm{a}}$ \\
\hline 3 & $99.313^{a}$ & $94.743^{a}$ & $95.74^{a}$ & $96.073^{a}$ & $92.193^{a}$ & $97.05^{\mathrm{a}}$ & $94.07^{a}$ & $91.487^{a b}$ & $89.15^{\mathrm{a}}$ & $83.863^{a}$ \\
\hline
\end{tabular}

*Los superíndices con la misma letra en las columnas no muestran diferencia significativa $(P \leq 0.05)$ de acuerdo a la prueba de Tukey. ND representa el dato inicial por lo tanto no hay valores. T1) quitosano puro al $2 \%$, T2) quitosano (2\%) + EAHM (1\%) y T3) Control (sin recubrimiento).

Tabla 11. Efecto de los tratamientos en variable luminosidad ( $\left.L^{*}\right)$.

Table 11. Effect of treatments on luminosity variable $\left(L^{*}\right)$.

\begin{tabular}{cccccccccccc}
\hline & \multicolumn{10}{c}{ Día } \\
\cline { 2 - 11 } Tratamiento & $\mathbf{1}$ & $\mathbf{2}$ & $\mathbf{3}$ & $\mathbf{4}$ & $\mathbf{5}$ & $\mathbf{6}$ & $\mathbf{7}$ & $\mathbf{8}$ & $\mathbf{9}$ & $\mathbf{1 0}$ \\
\hline $\mathbf{1}$ & $27.22^{\mathrm{a}}$ & $29.64^{\mathrm{a}}$ & $30.17^{\mathrm{a}}$ & $28.473^{\mathrm{a}}$ & $27.913^{\mathrm{a}}$ & $34.18^{\mathrm{a}}$ & $27.363^{\mathrm{a}}$ & $27.627^{\mathrm{a}}$ & $27.487^{\mathrm{a}}$ & $27.22^{\mathrm{a}}$ \\
$\mathbf{2}$ & $30.13^{\mathrm{a}}$ & $37.337^{\mathrm{a}}$ & $36.65^{\mathrm{a}}$ & $35.407^{\mathrm{a}}$ & $37.473^{\mathrm{a}}$ & $40.983^{\mathrm{a}}$ & $37.627^{\mathrm{a}}$ & $37.96^{\mathrm{a}}$ & $34.25^{\mathrm{a}}$ & $30.13^{\mathrm{a}}$ \\
$\mathbf{3}$ & $27.007^{\mathrm{a}}$ & $33.417^{\mathrm{a}}$ & $34.96^{\mathrm{a}}$ & $33.293^{\mathrm{a}}$ & $31.473^{\mathrm{a}}$ & $33.613^{\mathrm{a}}$ & $31.103^{\mathrm{a}}$ & $29.54^{\mathrm{a}}$ & $27.373^{\mathrm{a}}$ & $27.007^{\mathrm{a}}$ \\
\hline
\end{tabular}

* Los superíndices con la misma letra en las columnas no muestran diferencia significativa $(P \leq 0.05)$ de acuerdo a la prueba de Tukey. ND representa el dato inicial por lo tanto no hay valores. T1) quitosano puro al 2\%, T2) quitosano (2\%) + EAHM (1\%) y T3) Control (sin recubrimiento).

dos. Por otra parte, se observó DS $(P \leq 0.05)$ entre los días de evaluación. La relación tratamiento x tiempo no presentó interacción significativa $(P \leq 0.05)$ (tabla 4).

El cambio de color en los frutos frescos es un buen indicador del estado de maduración (Ribeiro et al., 2007). En este sentido, Han et al. (2004) y Perdones et al. (2012) señalaron que los recubrimientos de quitosano en fresa no afectan significativamente los valores Hue, observándose una disminución con respecto al paso de los días, atribuido a la síntesis de antocianinas; provocando una coloración roja oscura. Estos resultados son similares a los obtenidos en la presente investigación.

Los resultados de croma presentaron $D S(P \leq 0.05)$ los días 3, 4, 5, 6 y 8 (tabla 10). La tendencia de los tratamientos en el tiempo mostró DS $(P \leq 0.05)$, presentando cambios en los tratamientos durante los días de evaluación, con pendientes negativas, principalmente en el 1. Por otra parte, se observó $D S(P \leq 0.05)$ entre los días de evaluación. La relación tratamiento $\mathrm{x}$ tiempo presentó una interacción significativa $(P \leq 0.05)$ (tabla 4).

La disminución de los valores de croma obtenidos en la presente investigación es consistente con lo reportado por Hernández-Muñoz et al. (2006) quienes mencionaron una disminución significativa en los valores de croma en fresas recubiertas con quitosano después de 4 días. De igual manera, Ribeiro et al. (2007) señalaron que los recubrimientos de quitosano, almidón y carragenina en fresa provocaron un decremento en los valores de croma después de 6 días en almacenamiento. Esta disminución indica el oscurecimiento del color rojo típico de la fresa y es atribuido a la maduración. Por otra parte, Velickova et al. (2013) señalaron que los recubrimientos de quitosano con cera de abeja no indujeron cambios significativos en los valores de croma y Hue aplicados en fresas. Restrepo y Aristizábal (2010) indicaron que los recubrimientos de mucilago de penca de sábila y cera de carnaúba no afectaron los valores de croma en fresas. Estas diferencias en los resultados pueden estar relacionados con la variedad de la fresa, el estado de madurez, el tipo de recubrimiento y hasta las condiciones adversas a las que se hayan sometido las fresas antes del tratamiento.

Los resultados referentes a luminosidad $\left(L^{*}\right)$ no mostraron DS $(P \leq 0.05)$ en ninguno de los días evaluados (tabla 11). De igual forma, la tendencia en el tiempo del cambio de $L^{*}$ no mostró $D S(P \leq 0.05)$, presentando pendientes positivas al inicio del experimento y una caída en los últimos días. Por otra parte, se observó DS $(P \leq 0.05)$ entre los días de evaluación. La relación tratamiento $x$ tiempo presentó una interacción significativa $(P \leq 0.05)$ (tabla 4).

Duran et al. (2016) reportaron que los valores de luminosidad en fresas recubiertas con quitosano disminuyeron al final del tiempo de almacenamiento, sin embargo, no se observaron diferencias significativas. Además, señalaron que la incorporacione de agentes antimicrobianos no afectaron los valores de L*. De igual manera, Han et al. (2004) y TreviñoGarza et al. (2015) mencionaron que los recubrimientos de quitosano no tienen influencia en el color de las fresas en comparación con fresas sin recubrimientos. Estos resultados son similares a los reportados en el presente trabajo sin cambios significativos entre los 3 tratamientos en los días evaluados, lo que indica que el quitosano no afecta el oscurecimiento natural de la piel del fruto debido a procesos de oxidación y a la pérdida de humedad que se produce durante el almacenamiento (Restrepo y Aristizábal, 2010).

\section{CONCLUSIONES}

El método de extracción por ultrasonido y microondas es eficaz para evitar el uso de solventes y reducir el tiempo de extracción. Los recubrimientos aplicados mejoraron el 
índice de sabor y disminuyeron los valores de AT, mejorando así, la dulzura de la fresa. Sin embargo, no se observó mejora significativa en los parámetros de PP, pH y SST, con respecto al control.

\section{AGRADECIMIENTOS}

Los autores agradecen al programa "Apoyo a la incorporación de nuevo PTC-PRODEP 2015", F-PROMEP-38/ Rev-03, SEP-23-005. Además, un agradecimiento especial a la Universidad de Guanajuato por el apoyo mediante el proyecto CIFOREA 126/2016 y al Centro de Innovación y Desarrollo Agroalimentario de Michoacán (CIDAM) por el apoyo técnico y uso de sus instalaciones.

\section{REFERENCIAS}

Aday, M. S. and Caner, C. 2013. The shelf life extension of fresh strawberries using an oxygen absorber in the biobased package. LWT-Food Sci Technol. 52:102-109.

Ajavakom, A.; Supsvetson, S.; Somboot, A. and Sukwattanasinitt, M. 2012. Products from microwave and ultrasonic wave assisted acid hydrolysis of chitin. Carbohyd Polym. 90:73-77.

Alia-Tejacal, I.; Astudillo-Maldonado, Y. I.; Núñez-Colín, C. A.; Valdez-Aguilar, L. A.; Bautista-Baños, S.; García-Vázquez, E.; Ariza-Flores, R. y Rivera-Cabrera, F. 2012. Caracterización de frutos de ciruela mexicana (Spondias purpurea L.) del sur de México. Rev Fitotec Mex. 35:21-26.

Alvarado, Y.; Mendoza, R.; Sandoval, A. y Juárez, A. 2016. Comparación de diferentes sistemas de producción sobre crecimiento, rendimiento y calidad postcosecha de fresa Cv. San Andreas. Rev Csc Nat Agrop. 3:26-35.

AOAC. 1984. Official methods of analysis of the association of official analytical chemists (14th ed.). Washington, D. C.

Bagherian, H.; Zokaee, A. F.; Fouladitajar, A. and Mohtashamy, M. 2011. Comparisons between conventional, microwave- and ultrasound-assisted methods for extraction of pectin from grapefruit. Chem Eng Process. 50:1237-1243.

Chen, Y.; Gu, X.; Huang, S.; Li, J.; Wang, X. and Tang, J. 2010. Optimization of ultrasonic/microwave assisted extraction (UMAE) of polysaccharides from Inonotus obliquus and evaluation of its anti-tumor activities. Int J Biol Macromol. 46:429-435

Chien, P. J.; Sheu, F. and Yang, F. H. 2007. Effect of edible chitosan coating on quality and shelf life of sliced mango fruit. J Food Sci Eng. 78:225-229.

Chuang, P. H.; Lee, C. W.; Chou, J. Y.; Murugan, M.; Shieh, B. J. and Chen, H. M. 2007. Anti-fungal activity of crude extracts and essential oil of Moringa oleifera Lam. Bioresour Technol. 98:232-236.

Chumark, P.; Khunawat, P.; Sanvarinda, Y.; Phornchirasilp, S.; Phumala, M. N.; Phivthong-ngam, L.; Ratanachamnong, P.; Srisawat, S. and Pongrapeeporn, K. S. 2008. The in vitro and ex vivo antioxidant properties, hypolipidaemic and antiatherosclerotic activities of water extract of Moringa oleifera Lam. Leaves. J Ethnopharmacol. 116:439-446.

Cochran, W. G. and Cox, G. M. 1995. Diseños experimentales. 2a ed. (reimp.) Editorial Trillas, Ciudad de México, México. 661 pp.

Dahmoune, F.; Nayak, B.; Moussi, K.; Remini, H. and Madini, K. 2015. Optimization of microwave-assisted extraction of polyphenols from Myrtus communis L. leaves. Food Chem.166:585-595.

de Moraes, K. S.; Fagundes, C.; Melo, M. C.; Andreani, P. and Monteiro, A. R. 2012. Conservation of Williams pear using edible coating with alginate and carrageenan. Ciência Tecnol Alime. 32:679-684.

Dib, M. E. A.; Allali, H.; Bendiabdellah, A.; Meliani, N. and Tabti, B. 2013. Antimicrobial activity and phytochemical screening of Arbutus unedo L. J. Saudi Chem Soc. 17:381-385.

Duran, M.; Seckin, A. M.; Demirel, Z. N. N.; Temizkana, R.; Burak, B. M. and Caner, C. 2016. Potential of antimicrobial active packaging 'containing natamycin, nisin, pomegranate and grape seed extract in chitosan coating' to extend shelf life of fresh strawberry. Food Bioprod Process. 98:354-363.

Dutta, P.K.; Tripathi, S. and Methrota, G. K. 2008. Perspectives for chitosan based antimicrobial films in food application. Food Chem. 114:1173-1182.

Gol, N. B.; Patel, P. R. and Ramana, R. T. V. 2013. Improvement of quality and shelf-life of strawberries with edible coatings enriched with chitosan. Postharvest Biol Technol. 85:185195.

Gonzalez-Aguilar, G. A.; Monroy-Garcia, I. N.; GoycooleaValencia, F.; Diaz-Cinco, M. E. y Ayala-Zavala, J. F. 2005. Cubiertas comestibles de quitosano. Una alternativa para prevenir el deterioro microbiano y conservar la calidad de papaya fresca cortada. Nuevas tecnologías de conservación y envasado de frutas y hortalizas. 1:121-133.

Guzmán-Maldonado, S. H.; Zamarripa-Colmenares, A. y Hernández-Duran, L.G. 2015. Calidad nutrimental y nutraceutica de hoja de moringa proveniente de árboles de diferente altura. Rev Mex Cienc Agric. 6:317-330.

Han, C.; Zhaoa, Y.; Leonard, S. W. and Traber, M. G. 2004. Edible coatings to improve storability and enhance nutritional value of fresh and frozen strawberries (Fragaria $\times$ ananassa) and raspberries (Rubus ideaus). Postharvest Biol Technol. 33:67-78.

Hernández-Muñoz, P.; Almenar, E.; Ocio, M. O. and Gavara, R. 2006. Effect of calcium dips and chitosan coatings on postharvest life of strawberries (Fragaria $x$ ananassa). Postharvest Biol Technol. 39:247-253.

Huanga, L.; Cao, Y. and Chen G. 2008. Purification of quercetin in Anoectochilu roxburghii (wall) Lindl using UMAE by highspeed counter-current chromatography and subsequent structure identification. Sep Purif Technol. 64:101-107.

Jiang, Y. and Li, Y. 2001. Effect of chitosan coating on postharvest life and quality on longan fruit. Food Chem. 73:139-143.

Kasolo, J. N.; Bimenya, G. S.; Ojok, L.; Ochieng, J. and Ogwalokeng, J. W. 2010. Phytochemicals and uses of Moringa oleifera leaves in Ugandan rural communities. J Med Plants Res. 4:753-757.

Ladaniya, S. M. 2008. Citrus Fruits. Biology, Technology and Evaluation. Academic Press. San Diego, California, USA. 558 p.

Lianfu, Z. and Zelong, L. 2008. Optimization and comparison of ultrasound/microwave assisted extraction (UMAE) and ultrasonic assisted extraction (UAE) of lycopene from tomatoes. Ultrason Sonochem. 15:731-737.

Liew, S. Q.; Ngoh, G. C.; Yusoff, R. and Teoh, W. H. 2016. Sequential ultrasound-microwave assisted acid extraction (UMAE) of pectin from pomelo peels. Int J Biol Macromol. 93:426-435.

López-Mata, M.; Ruiz-Saul, S.; Navarro-Preciado, C.; Ornelas-Paz, J.; Estrado-Alvarado, M.; Gassos-Ortega, L. y Rodrigo-García, 
J. 2012. Efecto de recubrimientos comestibles de quitosano en la reducción microbiana y conservación de la calidad de las fresas. Biotecnia. 14:33-43.

Moyo, B.; Oyedemi, S.; Masika, P. J. and Muchenje, V. 2012. Polyphenolic content and antioxidant properties of Moringa oleifera leaf extracts and enzymatic activity of liver from goats supplemented with Moringa oleifera leaves/sunflower seed cake. Meat Sci. 91:441-447.

Oliveira, P. J.; Silva, G. C.; Alburquerque, C. R.; Lira de Sousa, F. J.; Fernandez, F. G.; Fonteles, F. A. and Silva dos Fernandes, V. R. 2011. In vitro antibacterial effect of aqueous and ethanolic Moringa leaf extracts. Asian Pac J Trop Med. 4:201-204.

Perdones, A.; Sánchez-González, L.; Chiralt, A. and Vargasa, M. 2012. Effect of chitosan-lemon essential oil coatings on storage-keeping quality of strawberry. Postharvest Biol Technol. 70:32-41.

Restrepo, F. J. y Aristizábal, T. I. 2010. Conservación de fresas (Fragaria $x$ ananassa Duch cv. Camarosa) mediante la aplicación de recubrimientos comestibles de gel mucilaginoso de penca de sábila (Aloe barbadensis Miller) y cera de carnúba. Vitae. 17:252-263.

Ribeiro, C.; Vicente A. A.; Teixeira, J. A. and Miranda, C. 2007. Optimization of edible coating composition to retard strawberry fruit senescence. Postharvest Biol Technol. 44:6370.

Rodríguez-Núñez, J. R.; López-Cervantes, J.; Sánchez-Machado, D. I.; Ramírez-Wong, B.; Torres-Chavez, P. and Cortez-Rocha, M. O. 2012. Antimicrobial activity of chitosan-based films against Salmonella typhimurium and Staphylococcus aureus. Int J Food Sci Tech. 47:2127-2133.

Rodríguez-Núñez, J. R.; Madera-Santana, T. J.; SánchezMachado, D.I.; López-Cervantes, J. and Soto-Valdez, H. 2014. Chitosan/Hydrophilic Plasticizer-Based Films: Preparation,
Physicochemical and Antimicrobial Properties. J Polym Environ. 22:41-51.

SAGARPA. (2017). Avance de siembras y cosechas. Resumen nacional por estado. Disponible en: http://infosiap.siap.gob. mx:8080/agricola_siap_gobmx/AvanceNacionalCultivo.do

Singleton, V. L. and Rossi, J. A. 1965. Colorimetric of total phenolics with phosphomolibdic-phosphotungstic acid reagents. Am J Enol Vitic. 16:144-58.

Suyatma, E.;Tighzert, L. and Copinet, A. 2005. Effect of hydrophilic plasticizers on mechanical, thermal, and surface properties of chitosan films. J Agric Food Chem. 53:3950-3967.

Treviño-Garza, M. Z.; García, S.; Flores-González, M. S. and Arévalo-Niño, K. 2015. Edible Active Coatings Based on Pectin, Pullulan, and Chitosan Increase Quality and Shelf Life of Strawberries (Fragaria ananassa). J Food Sci. 80:M1823.

Velickova, E.; Winkelhausen, E.; Kuzmanova, S.; Alves, V. D. and Moldão-Martins, M. 2013. Impact of chitosan-beeswax edible coatings on the quality of fresh strawberries (Fragaria ananassa cv Camarosa) under commercial storage conditions. LWT Food Sci Technol. 52:80-92.

Verma, A. R.; Vijayakumar, M.; Mathela, C. S. and Rao, C. V. 2009. In vitro and in vivo antioxidant properties of different fractions of Moringa oleifera leaves. Food Chem Toxicol. 47:2196-2201.

Wang, Y.; Li, R.; Jiang Z-T.; Tan J.; Tang S-H.; Li T-T.; Liang L-L.; He H-J.; Liu Y-M.; Li J-T. and Zhang X-G. 2018. Green and solventfree simultaneous ultrasonic-microwave assisted extraction of essential oil from white and black peppers. Ind Crops Prod. 114: 164-172.

Zhang, D. L. and Quantick, P. C. 1997. Effect of chitosan coating enzymatic browning and decay during postharvest storage of litchi (Litchi chinensis Sonn) fruit. Postharvest Biol Technol. 12: 195-202. 